\title{
The Strange Quark Polarisation from Charged Kaon Production on Deuterons
}

\author{
R.Windmolders (On behalf of the COMPASS collaboration) \\ Universität Bonn, Physikalisches Institut, 53115 Bonn, Germany
}

\begin{abstract}
The strange quark helicity distribution $\Delta s(x)$ is derived at LO from the semi-inclusive and inclusive spin asymmetries measured by the COMPASS experiment at CERN. The significance of the results is found to depend critically on the ratio of the $\bar{s}$ and $u$ quark fragmentation functions into kaons ${ }^{\mathrm{R}} D_{\bar{s}}^{K+}(z) d z={ }^{\mathrm{R}} D_{u}^{K+}(z) d z$.
\end{abstract}

Keywords: Deep inelastic scattering; Polarised parton densities

PACS: $13.60 . \mathrm{Hb} ;$ 13.60.Le; 13.88.+e

In contrast to other sea quarks, the strange quark contribution to the nucleon spin is accessible in inclusive lepton-nucleon scattering experiments. Already twenty years ago the EMC [1] has found its first moment $\Delta s+\Delta \bar{s}$ to be negative under the assumption of $\mathrm{SU}(3)_{F}$ symmetry in hyperon $\beta$ decays and this result has been confirmed with improved precision by recent measurements performed by HERMES [2] $(\Delta s+\Delta \bar{s}=$ $0: 1030007$ (exp :) $0: 013$ (theor :) $0 \$ 008$ (evol :)) and by COMPASS [3]:

$$
\Delta s+\Delta \bar{s}=0.09 \quad 0.01 \text { (stat:) } \quad 0.02 \text { (syst:): }
$$

Inclusive experiments provide a direct evaluation of the first moment of $(\Delta s+\Delta \bar{s})$ only. However the distribution $\Delta s\left(x ; Q^{2}\right)$ can be obtained from semi-inclusive channels in which interactions on strange quarks are favoured, such as charged kaon production. These measurements require final state particle identification and became only feasible in recent experiments $[4,5]$.

In this paper we present a leading order (LO) evaluation of the polarised parton distributions $\Delta u_{v}+\Delta d_{v}, \Delta \bar{u}+\Delta \bar{d}$ and $\Delta s(=\Delta \bar{s})$ derived from the inclusive and semiinclusive spin asymmetries measured by the COMPASS experiment. The data sample covers the years 2002-2004 and 2006, and corresponds to the full statistics collected on the longitudinally polarised ${ }^{6} \mathrm{LiD}$ target exposed to the $160 \mathrm{GeV}$ muon beam at CERN. The averaged beam and target polarisations were $0: 80$ and 050 , respectively. Each selected event contains a beam muon with energy $140<E_{\mu}<180 \mathrm{GeV}$, a scattered muon and, for the semi-inclusive samples, at least one charged hadron originating from the interaction vertex. The DIS conditions $Q^{2} \quad 1(\mathrm{GeV}=c)^{2}$ and $0: 1 \quad$ y $\quad 0.9$ are required in the muon kinematics. The hadron is required to carry a fractional energy $0: 20<z<0: 85$ and must be identified as a pion or a kaon by the RICH detector, which limits its momentum to the range $10<p<50 \mathrm{GeV} / \mathrm{c}$. The total number of identified hadrons is $2310 \pi^{+}, 2116 \pi, 4: 816 \mathrm{~K}^{+}$and $3: 310 \mathrm{~K}$. The purity of the selected samples is above $95 \%$ for the pions and between 80 and $90 \%$ for the kaons.

The inclusive and semi-inclusive asymmetries are shown in Fig. 1 as a function of the scaling variable $x$ in a range limited by the kinematical threshold $Q^{2} \quad 1(\mathrm{GeV}=c)^{2}$, 
which implies $x>0004$, and a value where sea quarks distributions become insignificant $(x<0: 3)$.

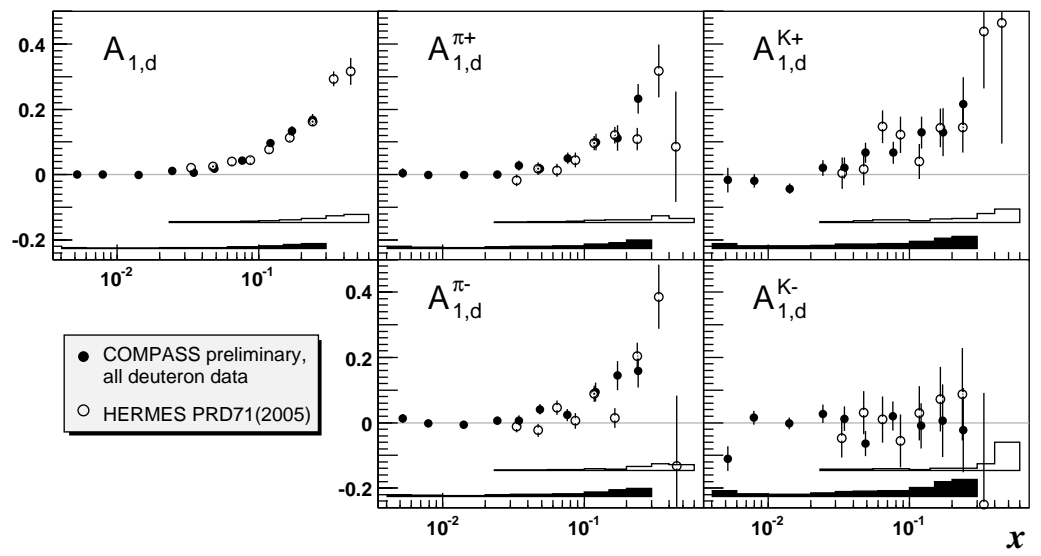

FIGURE 1. Spin asymmetries of COMPASS compared to the HERMES results [4]. The bands show the systematic uncertainties for the two sets of data.

The correction applied to account for the contamination of the pion and kaon samples has only a small effect on the semi-inclusive asymmetries. The HERMES data [4], shown on the same plots, are in good agreement with the present ones in the region of overlap and their quoted errors are generally also comparable. The COMPASS systematic error includes an overall scale uncertainty due to the uncertainties on the beam and target polarisation, on the dilution factor and on the depolarisation factor which amount to $8 \%$ when added in quadrature. In addition, each point has an uncertainty due to possible apparatus induced asymmetries which are estimated to be less than $0.4 \sigma$ (stat:).

The polarised parton densities (PDFs) have been evaluated at $Q_{0}^{2}=3(\mathrm{GeV}=c)^{2}$ in each bin of $x$ by a least square fit on the asymmetries assumed to be independent of $Q^{2}$ (Fig. 2). In this calculation, unpolarised PDFs are taken from the MRST parameterisation [6] and fragmentation functions (FFs) from the recent DSS parameterisation [7], both at LO. In order to test the dependence of the polarised PDFs on the FFs, we also show the values obtained with the EMC FFs [8]. In contrast to other parameterisations which are derived from global fits, the latter ones have been extracted from the EMC data only, so that only the $u$ quark fragmentation could be measured. Therefore, in addition to the usual assumptions made to reduce the number of FFs, it was also assumed that $D_{\bar{s}}^{K^{+}}=D_{u}^{\pi+}$.

The comparison of the two sets of values shows that the valence and non-strange sea distributions depend very little on the choice of FFs while $\Delta s(x)$ and its statistical error increase by a factor of 2-3 when the DSS FFs are replaced by the EMC ones. The valence distribution is in good agreement with the DNS parameterisation [9] and with a previous evaluation based on the difference asymmetry for non-identified hadrons $A^{h^{+}} h^{h}$ [3]: its first moment truncated to the measured range is $0.28 \quad 0: 06$ (stat:) 0.03 (syst :), vs. $0.26 \quad 0.07 \quad 0.04$ at $Q^{2}=10(\mathrm{GeV} / \mathrm{c})^{2}$ in Ref. [3]. The non-strange sea distribution is consistent with zero for all values of $x$. The strange quark distribution obtained with the DSS FFs is also consistent with zero and its truncated moment is $\quad 0.01 \quad 0.01 \quad 0.01$ (vs. $\quad 0: 05 \quad 0: 03 \quad 0: 01$ with the EMC FFs). 


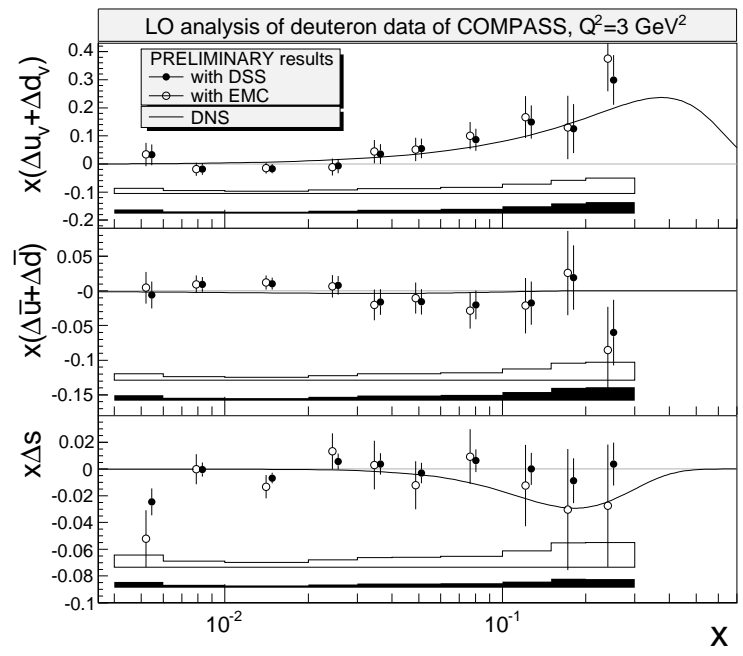

FIGURE 2. The quark helicity distributions evaluated at $Q_{0}^{2}=3(\mathrm{GeV}=c)^{2}$ as a function of $x$ for two sets of fragmentation functions (DSS [7] and EMC[8]). The bands show the corresponding systematic uncertainties. The curves represent the LO DNS parameterisation of polarised PDFs [9].

The dependence of $\Delta s(x)$ on the FFs can be further explored by introducing the charged kaon asymmetry

$$
A^{K^{+}+K}={ }^{K^{+}} A^{K^{+}}+\sigma^{K} A^{K} \stackrel{\text { i h }}{=} \sigma^{K^{+}}+\sigma^{K} \stackrel{\text { i }}{:}
$$

These asymmetries are found to be very stable with respect to changes in the cross-section ratio $\sigma^{K}=\sigma^{K^{+}}$. At LO this ratio only depends on unpolarised PDFs and on the ratios of unfavoured to favoured and strange to favoured FFs: $R_{U F}={ }^{\mathrm{R}} D_{d}^{K^{+}}(z) d z={ }^{\mathrm{R}} D_{u}^{K^{+}}(z) d z$ and $R_{S F}={ }^{\mathrm{R}} D_{\bar{s}}^{K^{+}}(z) d z={ }^{\mathrm{R}} D_{u}^{K^{+}}(z) d z$, which are equal to 0.14 and 6.6 for the DSS FFs at $Q^{2}=3(\mathrm{GeV}=c)^{2}(0.35$ and 3.4 for the EMC FFs $)$. The values shown in Fig. (3, left) have been obtained with the MRST PDFs and the DSS FFs. As for the $K^{+}$and $K$ asymmetries, they are in very good agreement with the HERMES values of Ref. [4].

The strange quark polarisation is related to $A^{K^{+}+K}$ and to the inclusive asymmetry $A_{1}^{d}$ by the linear relation

$$
\frac{\Delta s}{s}=A_{1}^{d}+\left(A^{K^{+}+K} \quad A_{1}^{d}\right) \frac{Q=s+\alpha}{\alpha \quad 08}
$$

where $Q=u+\bar{u}+d+\bar{d}$ is the non-strange quark density and $\alpha=\left(2 R_{U F}+\right.$ $\left.2 R_{S F}\right)=\left(3 R_{U F}+2\right)$. As expected, the use of the above formula with the $A^{K^{+}+K}$ values of Fig. 3 leads to values of $\Delta s$ practically equal to those of Fig. 2, with slightly larger statistical errors. Several other interesting features can be derived from Eq.(3). In the special case where $A^{K^{+}+K}=A_{1}^{d}, \Delta s$ becomes insensitive to the FFs and its first moment is very small and positive. Otherwise the main dependence on the FFs is due to $R_{S F}$ which appears only in the numerator of $\alpha$, and its effect is amplified by the 
large values of the ratio $Q=S$. At low $x$ where $A_{1}^{d} \quad 0$, negative values of $A^{K^{+}+K}$ imply $\Delta s<0$. The COMPASS values of $A^{K^{+}+K}$ at low $x$ provide at least a hint that $\Delta s$ may become negative in the previously unmeasured region $x<0.02$, as predicted in the recent DSSV fit [10].
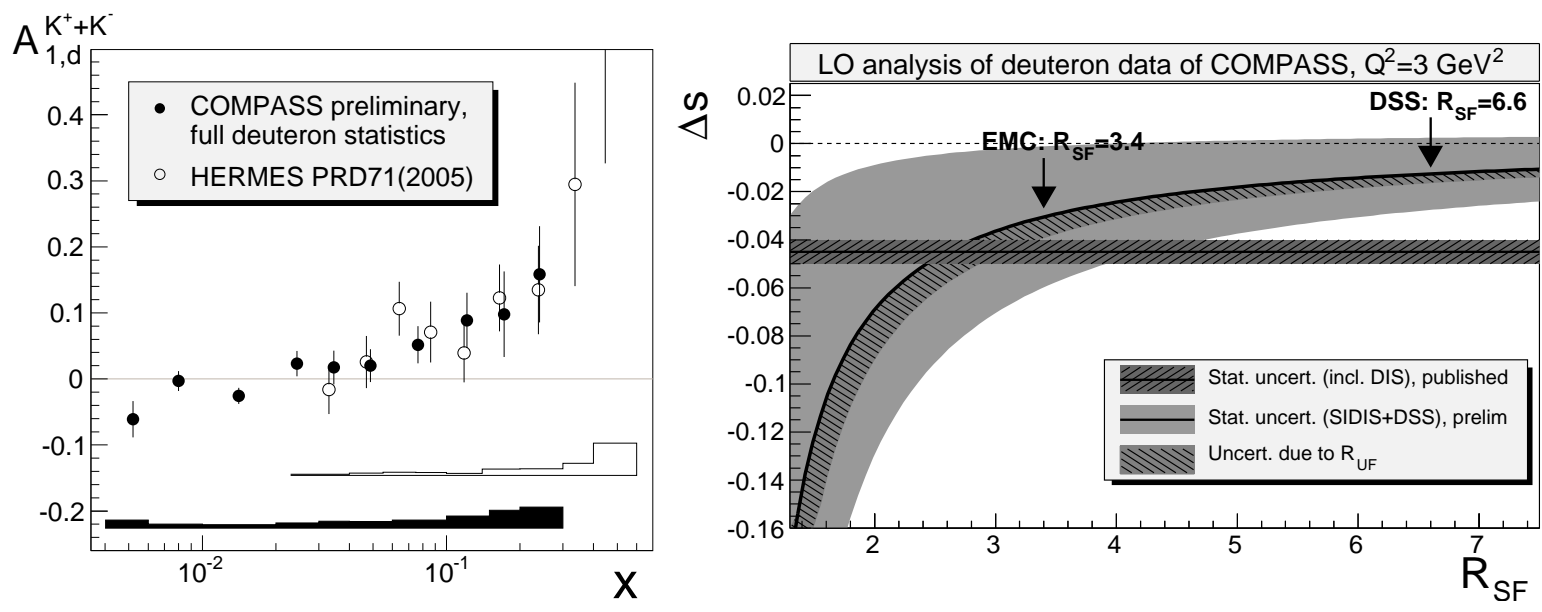

FIGURE 3. Left: Charged kaon asymmetries obtained with cross-section weights from MRST PDF's and DSS FF's. Right: Integral of $\Delta s$ over the measured range of $x$, as a function of the ratio $R_{S F}$ for $R_{U F}$ fixed at the DSS value of 0.14 (thick solid curve). The light-gray area shows the statistical uncertainty and the hatched band inside of it shows the effect of increasing $R_{U F}$ to 0.35 (EMC value). The horizontal band represents the full moment of $\Delta s$ derived from the COMPASS value of $\Gamma_{1}^{N}$ (Eq. 1).

Fig. (3, right) shows the variation of the first moment of $\Delta s$ truncated to the measured region as a function of $R_{S F}$. For $R_{S F} \& 5$, we observe that the values are close to zero and larger than the full moment derived from the inclusive analy sis (Eq.1). In particular this is the case for the DSS FFs where $R_{S F}=6.6$. The difference never exceeds two standard deviations so that no firm conclusions can be drawn from the COMPASS data alone but, as shown on Fig. (3, left) the HERMES data lead to a similar result. In contrast, if $R_{S F}$. $4, A^{K^{+}+K}$ becomes less and less sensitive to $\Delta s$ because $D_{\bar{s}}^{K^{+}}$is small. The conclusions on $\Delta s$ obtained in the present analysis are thus conditional on the ratio $R_{S F}$ which will later be derived from the $K^{+}$and $K$ rates observed in the COMPASS data.

\section{REFERENCES}

1. EMC, J. Ashman, et al., Phys. Lett. B 206, 364-370 (1988).

2. HERMES Collaboration, A. Airapetian, et al., Phys. Rev. D 75, 012007, 1-48 (2007).

3. COMPASS Collaboration, M. Alekseev, et al., Phys. Lett. B 660, 458-465 (2008).

4. HERMES Collaboration, A. Airapetian, et al., Phys. Rev. D 71, 012003, 1-36 (2005).

5. HERMES Collaboration, A. Airapetian, et al., arXiv:0803.2993vl (hep-ex),20 Mar 2008.

6. A. D. Martin, W. J. Sterling, R. S. Thorne, Phys. Lett. B 636, 259-264 (2006).

7. D. de Florian, et al., Phys. Rev. D 75, 114010, 1-26 (2007).

8. EMC, M. Arneodo, et al., Nucl. Phys. B 321, 541-560 (1989).

9. D. de Florian, G. A. Navarro, R. Sassot, Phys. Rev. D 71, 094018, 1-12 (2005).

10. D. de Florian, R. Sassot, M. Stratmann, W. Vogelsang, Phys. Rev. Lett. 101, 072001, 1-4 (2008). 